\title{
WHSCl acts as a prognostic indicator and functions as an oncogene in cervical cancer
}

This article was published in the following Dove Press journal: OncoTargets and Therapy

\author{
Jin $\mathrm{Wu}$ \\ Ming Luo \\ Zhaoning Duan \\ Ying Jia \\ Hua Linghu \\ $\mathrm{Pu}$ Tian \\ Hongbo Qi
}

Obstetrics and Gynecology of the First Affiliated Hospital of Chongqing Medical University, Chongqing, People's Republic of China
Correspondence: Hongbo Qi

Obstetrics and Gynecology of the First Affiliated Hospital of Chongqing Medical University, Youyi Road No. I, Yuzhong

District, Chongqing 4000 16, People's

Republic of China

Tel +86 I38 08376 I I6

Fax +862389011082

Email qihb2018@I63.com
Purpose: Wolf-Hirschhorn syndrome candidate 1 (WHSC1) is an epigenetic modifier, considered to play a driving role in oncogenesis. However, very little is known about the roles of WHSC1 and its prognostic impacts in cervical cancer. This study aimed to investigate the role of WHSC1 in the prognosis of cervical cancer and explore the effect of WHSC1 on proliferation, migration, and invasion of cervical cancer cells and angiogenesis in human umbilical vein endothelial cells (HUVECs).

Methods: We evaluated the expression levels of WHSC1 in cervical cancer samples and relevant cell lines by immunohistochemistry, real-time quantitative PCR, and Western blot. In vitro, Cell Counting Kit- 8 and transwell assays were used to investigate the viability and migration of C33A cells, and a tube formation assay was used to study the effect of WHSC1 on angiogenesis in HUVECs.

Results: WHSC1 was overexpressed in cervical cancer tissues and cells, and correlated with the FIGO stage and differentiation. WHSC1 knockdown inhibited proliferation, suppressed migration and invasion in endothelial nitric oxide synthase (eNOS)-overexpressing C33A cells, and inhibited angiogenesis in HUVECs.

Conclusion: WHSC1 may be a poor prognostic indicator of cervical cancer and a potential novel therapeutic target for the same. WHSC1 may participate in the regulation of cervical cancer progression through the eNOS signaling pathway.

Keywords: Wolf-Hirschhorn syndrome candidate 1, cervical cancer, growth, angiogenesis, endothelial nitric oxide synthase

\section{Introduction}

Invasive cervical cancer (CC) is the fourth most common form of cancer worldwide and the second most common in developing countries. ${ }^{1}$ Despite advancements in radiotherapy and surgical therapy, numerous patients with late-stage CC still experience metastasis and recurrence, particularly in developing countries. ${ }^{2}$ Hence, sincere efforts are being directed toward the development of effective targeted therapies for CC.

Epigenetic modifiers are widely recognized as targets for therapeutic intervention, owing to their critical roles in regulating gene expression and chromatin integrity in addition to their dysregulation in a range of human pathologies. ${ }^{3}$ In particular, the nuclear receptor-binding SET domain NSD2 family of histone lysine methyltransferase enzymes, namely NSD1, NSD2/WHSC1/MMSET, and NSD3/WHSC1L1, which are involved in developmental and tumoral pathologies, ${ }^{4}$ have all been implicated as cancer therapeutic targets. ${ }^{5}$ The oncogenic role of Wolf-Hirschhorn syndrome candidate 1 (WHSC1) was first reported in multiple myeloma, in which the $(4 ; 14)(\mathrm{p} 16$; 
q32) translocation results in WHSC1 overexpression. ${ }^{6-8}$ WHSC1 is also frequently overexpressed in solid tumors, such as in cases of oligodendroglioma, breast, prostate, and head and neck cancers. ${ }^{9-11}$ Nevertheless, the genetic characterization of $\mathrm{WHSC} 1$, to determine its role in CC metastasis, and signaling coordinated by WHSC1 remain undefined.

To determine the expression pattern of WHSC1 in $\mathrm{CC}$, we profiled the expression status of WHSC1 in various cervical tissues. Moreover, we analyzed the association between WHSC1 expression and clinicopathological characteristics of CC. After examining the effect of WHSC1 on the biological function of C33A cells and human umbilical vein endothelial cells (HUVECs) in vitro, we want to reveal the possible role of WHSC1 in the development and angiogenesis of $\mathrm{CC}$.

\section{Materials and methods}

\section{Patients and tissue samples}

$\mathrm{CC}$ and cervical intraepithelial neoplasia tissues were collected from 84 patients (tissue samples for cervical biopsy were punched based on findings from colposcopy) and normal cervical samples were collected from 12 patients who underwent hysterectomy for hysteromyoma between May 2013 and November 2014 at the First Affiliated Hospital of Chongqing Medical University, People's Republic of China. None of these patients had received adjuvant therapy until cervical biopsy. All tissue specimens were snap-frozen immediately in liquid nitrogen after harvest, and stored at $-80{ }^{\circ} \mathrm{C}$. This study was approved by the Ethics Committee of the First Affiliated Hospital of Chongqing Medical University, and written informed consent was obtained from all patients. All experiments were performed in accordance with relevant guidelines and regulations.

\section{Construction of tissue microarrays (TMAs) and immunohistochemistry}

Cervical tissue TMAs were prepared as described previously. Two-millimeter core TMAs were constructed from each of the collected cervical tissues that were marked by pathologists in Chongqing Medical University. Immumohistochemistry analyses were performed using anti-WHSC1 (ab137429; Abcam) antibodies. Immunohistochemical staining was performed on TMA sections according to the manufacturer's protocol. We used a metastatic carcinoma with high expression of
WHSC1 as a positive control in immunohistochemistry. Protein expression was scored and quantified by two independent pathologists blinded to the outcome of the cases.

\section{Cell culture}

$\mathrm{CC}$ cell lines SIHA and C33A were maintained in minimal essential medium, supplemented by $10 \%$ FBS, $1 \%$ penicillin/streptomycin, and $1 \%$ Glutamax. HUVECs and HELA cells were maintained in DMEM medium, containing $10 \%$ FBS, $1 \%$ penicillin/streptomycin, and $1 \%$ Glutamax. Human embryonic kidney (HEK) cells were maintained in DMEM/F12K medium, with $10 \% \mathrm{FBS}$, $1 \%$ penicillin/streptomycin, and $1 \%$ Glutamax. All cells were purchased from the cell bank of the Chinese Academy of Sciences.

\section{Lentiviral vector and transfection}

Lenti-WHSC1-EGFP-mir vector and small interfering RNAs (siRNAs) were obtained from Guangzhou Ribobio Biotechnology, Guangzhou, People's Republic of China. The siRNA sequences targeting the WHSC1 gene are listed in Table S1. C33A cells were either transfected with Lenti-WHSC1-EGFP-mir or Lenti-EGFP, or siRNAs targeting WHSC1 (Ribobio, Guangzhou, People's Republic of China). The knockdown or overexpression efficiency was evaluated by fluorescence microscopy, quantitative reverse transcription PCR (qRT-PCR), and Western blot analysis.

\section{Cell proliferation assay}

Cell Counting Kit-8 (CCK-8) (Sigma-Aldrich Co., St Louis, MO, USA) was used to determine cell proliferation. Briefly, C33A cells were seeded onto 6-well plates before treatment with siWHSC1 or siNC, and grown to about $75 \%$ confluence over $24 \mathrm{~h}$ at $37{ }^{\circ} \mathrm{C}$. Post treatment with siWHSC1 or siNC for $0,24,48,72,96,120$, and $144 \mathrm{~h}$, cells were washed thrice with PBS and $100 \mu \mathrm{L} /$ well of PBS was added to each well. Next, $10 \mu \mathrm{L}$ of the CCK-8 working solution (CK04; Dojindo) was added to each well. After incubation at $37{ }^{\circ} \mathrm{C}$ for $150 \mathrm{~min}$, the fluorescence intensity of each well was recorded at $450 \mathrm{~nm}$ using a fluorescence microplate reader (BioTek, Winooski, VT, USA).

\section{Cell migration and invasion assays}

Cell migration and invasion ability were analyzed using cell migration assay kits (R\&D Systems Inc., Minneapolis, $\mathrm{MN}$, USA) following the manufacturer's protocols. 


\section{Tube formation assay}

At first, 96-well plates were coated with Geltrex Reduced Growth Factor Basement Membrane Matrix (Invitrogen; Thermo Fisher Scientific, Waltham, MA, USA) $(50 \mu \mathrm{L} /$ well) and incubated at $37{ }^{\circ} \mathrm{C}$ for $30 \mathrm{~min}$ to allow gelation to occur. HUVECs were added on top of the gel at a density of 50,000 cells/wells and cultured in M200 medium containing $50 \mu \mathrm{L}$ conditioned medium freshly collected from C33A cells subjected to Lentivirus transfection for $6 \mathrm{~h}$. After incubation for $6 \mathrm{~h}$, tube formation was monitored and photographed under a phase-contrast microscope (Olympus Corporation, Tokyo, Japan). Tubelike structures exceeding 3 joint points were counted.

\section{Western blot}

Cells were lysed with $100 \mu \mathrm{L}$ loading buffer, boiled for 10 min, centrifuged at 12,000 rpm for $1 \mathrm{~min}$, and subsequently stored at $-20{ }^{\circ} \mathrm{C}$. The proteins were separated by SDSPAGE and transferred to polyvinylidene difluoride membranes. The membrane was blocked with $5 \%$ nonfat dried milk in TBST solution at room temperature (about $25^{\circ} \mathrm{C}$ ) for $1 \mathrm{~h}$ and incubated with primary antibodies at $4{ }^{\circ} \mathrm{C}$ overnight. The following primary antibodies were used: WHSC1 (1:500; Abcam), p-AKT (1:1,000, 4060; CST), endothelial nitric oxide synthase (eNOS) (1:1,000, ab76198; Abcam), and actin (1:10,000; SunGene Biotech). The membrane was then incubated with anti-rabbit or anti-mouse or anti-goat secondary antibody conjugated with horseradish peroxidase for $1 \mathrm{~h}$ at room temperature. Finally, the protein bands were detected by enhanced chemiluminescence (EMD Millipore, Billerica, MA, USA) and band intensities were analyzed by ImageJ software.

\section{Reverse transcription-polymerase chain reaction (RT-PCR)}

The target mRNAs in cells and tissues were assessed by the RT-PCR method. The total RNA of cells and tissues was extracted using TRIzol ${ }^{\circledR}$ reagent (Thermo Fisher Scientific) according to the manufacturers' instructions. $\beta$-actin was used as an internal control. qRT-PCR reactions were carried out using the SYBR Green One-Step RT-qPCR kit (Thermo Fisher Scientific) according to the manufacturer's instructions. The primer sequences are detailed in Table S2.

\section{Statistical analysis}

All experiments were performed independently at least thrice. All data were analyzed using SPSS 20.0 software (IBM Corporation, Armonk, NY, USA). The $\chi 2$ test was used to explore the association between WHSC1 expression and clinicopathological variables. Kaplan-Meier curves and the log-rank test were used for the analysis of survival data. For cellular experiments, all data were expressed as mean $\pm \mathrm{SD}$ of 3 independent experiments performed in triplicate. Statistical significance was assessed with Student's $t$-test or oneway analysis of variance. $P<0.05$ was considered statistically significant.

\section{Results}

\section{Overexpression of $\mathrm{WHSCl}$ in patients} with cervical cancer and correlation with clinicopathological parameters

To investigate the expression of WHSC1 in CC, immunohistochemistry staining was performed in a TMA containing 96 cervical samples, including cervicitis (18 cases, $18.8 \%$ ), cervical intraepithelial neoplasia 3 (6 cases, $6.3 \%$ ), and CC (72 cases, 75\%). Thereafter, immunostaining grade analysis was performed based on both the proportion of positively stained cells and the intensity of staining (Figure 1A). Characteristics of patients with $\mathrm{CC}$ are shown in Table 1 . The density of WHSC1 was significantly higher in cases of late cancer stage $(P=0.0040)$ and poor differentiation $(P=0.0156)$; however, it was not significantly higher according to tumor size $(P=0.4999)$, lymph node metastasis $(P=0.5131)$, and patients' age $(P=0.5929)$. WHSC1 mRNA and protein expression levels were more highly expressed in the tumor tissues of patients with $\mathrm{CC}(P<0.05)$ (Figure 1B) than in normal tissues. The Kaplan-Meier survival curves indicated that patients with $\mathrm{CC}$, having high WHSC1 expression, had poorer overall survival rates than those with low expression (log-rank, $P=0.0250$, HR: 2.164, 95\% CI: 1.102 to 4.25) (Figure 1C).

\section{Overexpression of WHSCl in C33A cells and inhibition by siRNAs}

HELA, SIHA, and C33A cells were used to investigate WHSC1 expression, and HEK cells were used as the control. Results showed that the mRNA and protein levels of WHSC1, eNOS, and AKT were significantly higher in C33A and SIHA cells than in HEK cells (Figure 2). There was no significant difference between the expression of WHSC1 mRNA in HELA and HEK cells. In addition, 


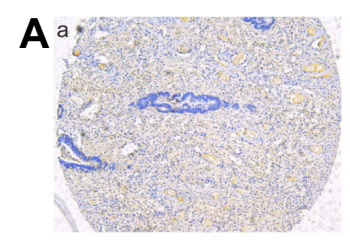

B

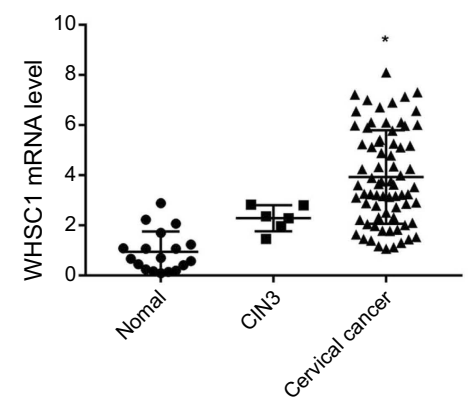

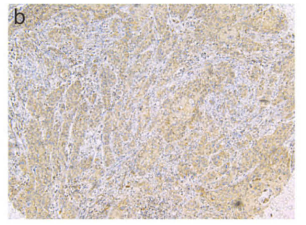
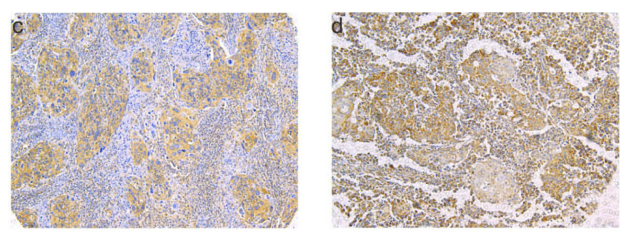

C

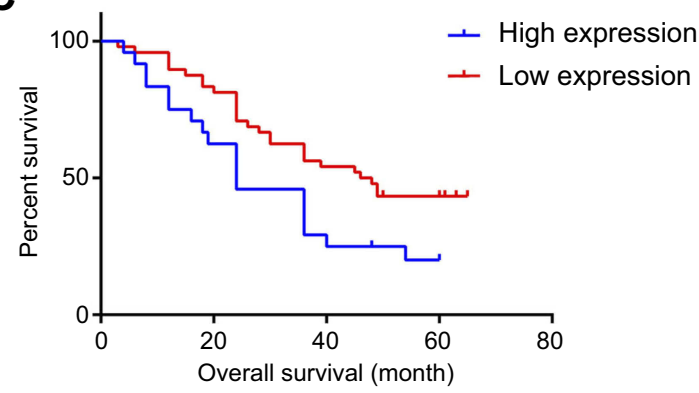

Figure I WHSCI expression in CC samples. (A) WHSCI expression in tissue microarrays of CC tissue: (a) normal cervical tissues with no or weak WHSCI expression were read negative; (b) low expression of WHSCI in CC tissues was +; (c) medium expression of WHSCI in CC tissues was ++; (d) high expression of WHSCI in CC tissues was +++ . (B) WHSCI mRNA levels in $C$ C tissues. ${ }^{*} P<0.05$ vs normal tissues or $\mathrm{CIN} 3$. (C) Kaplan-Meier survival curves of patients with $C \mathrm{C}$, based on WHSCI expression status.

Abbreviations: CC, cervical cancer; WHSCI, Wolf-Hirschhorn syndrome candidate I.

Table I Correlations of WHSCI expression with clinicopathological parameters of patients with cervical cancer

\begin{tabular}{|l|l|l|l|l|}
\hline \multirow{2}{*}{$\begin{array}{l}\text { Clinicopathological } \\
\text { parameter }\end{array}$} & \multirow{2}{*}{$\begin{array}{l}\text { Case } \\
\text { number }\end{array}$} & \multicolumn{2}{|l|}{ WHSCI } & \multirow{2}{*}{ P-value } \\
\cline { 3 - 4 } & & Low & High & \\
\hline Total & 72 & 52 & 20 & \\
\hline Age (years) & & & & \\
$<50$ & 44 & 33 & 11 & 0.5929 \\
$\geq 50$ & 28 & 19 & 9 & \\
\hline Tumor size (cm) & & & & \\
$<4$ & 58 & 44 & 14 & 0.4999 \\
$\geq 4$ & 14 & 9 & 5 & \\
\hline Nodal involvement & & & & \\
Yes & 14 & 9 & 5 & 0.5131 \\
No & 58 & 43 & 15 & \\
\hline Differentiation & & & & \\
Well/moderately & 60 & 47 & 13 & $0.0156 *$ \\
Poorly & 12 & 5 & 7 & \\
\hline Stage & & & & \multirow{2}{*}{$0.0040^{* *}$} \\
I & 2 & 2 & 0 & \\
II & 28 & 26 & 2 & \\
III & 42 & 25 & 18 & \\
\hline Notes: & & & \\
\hline
\end{tabular}

Notes: $* P<0.05, * * P<0.005 .+,++$ and +++ represent the intensity of the expression of $\mathrm{WHSCl}$, respectively, with detailed annotations in Figure IA. + and ++ were named low and medium expressions, +++ was high expression.

Abbreviation: WHSCI, Wolf-Hirschhorn syndrome candidate I.

both mRNA and protein expression levels of WHSC1 were inhibited significantly by siWHSC1-3\# in C33A cells compared to that in NC cells (Figure 2).

\section{siWHSCI inhibits proliferation and suppresses migration and invasion of C33A cells}

To determine the functional role of WHSC1 in cell proliferation, we performed the CCK- 8 assay. siWHSC1 treatment significantly inhibited the viability of C33A cells compared to that of control cells (Figure 3). We performed transwell assays to investigate the effects of WHSC1 on C33A cell mobility. Results revealed that the invasion abilities of C33A cells after siWHSC1 treatment are remarkably reduced compared to that of untreated cells (Figure 3).

\section{siWHSCI inhibited angiogenesis in HUVECs}

An in vitro tube formation assay, based on HUVECs, was used to observe angiogenesis in C33A cells. HUVECs treated with the conditioned medium of $\mathrm{C} 33 \mathrm{~A} / \mathrm{siWHSC} 1$ cells were significantly inhibited from forming extensive and enclosed tube networks, compared to untreated cells $(P<0.05$, Figure 4A).

\section{WHSCI knockdown results in increased eNOS expression}

To examine whether WHSC1 affects eNOS expression in CC, C33A cells were transfected with a Lenti-WHSC1EGFP-mir vector. The protein expression of eNOS in C33A cells was significantly enhanced by reduced 

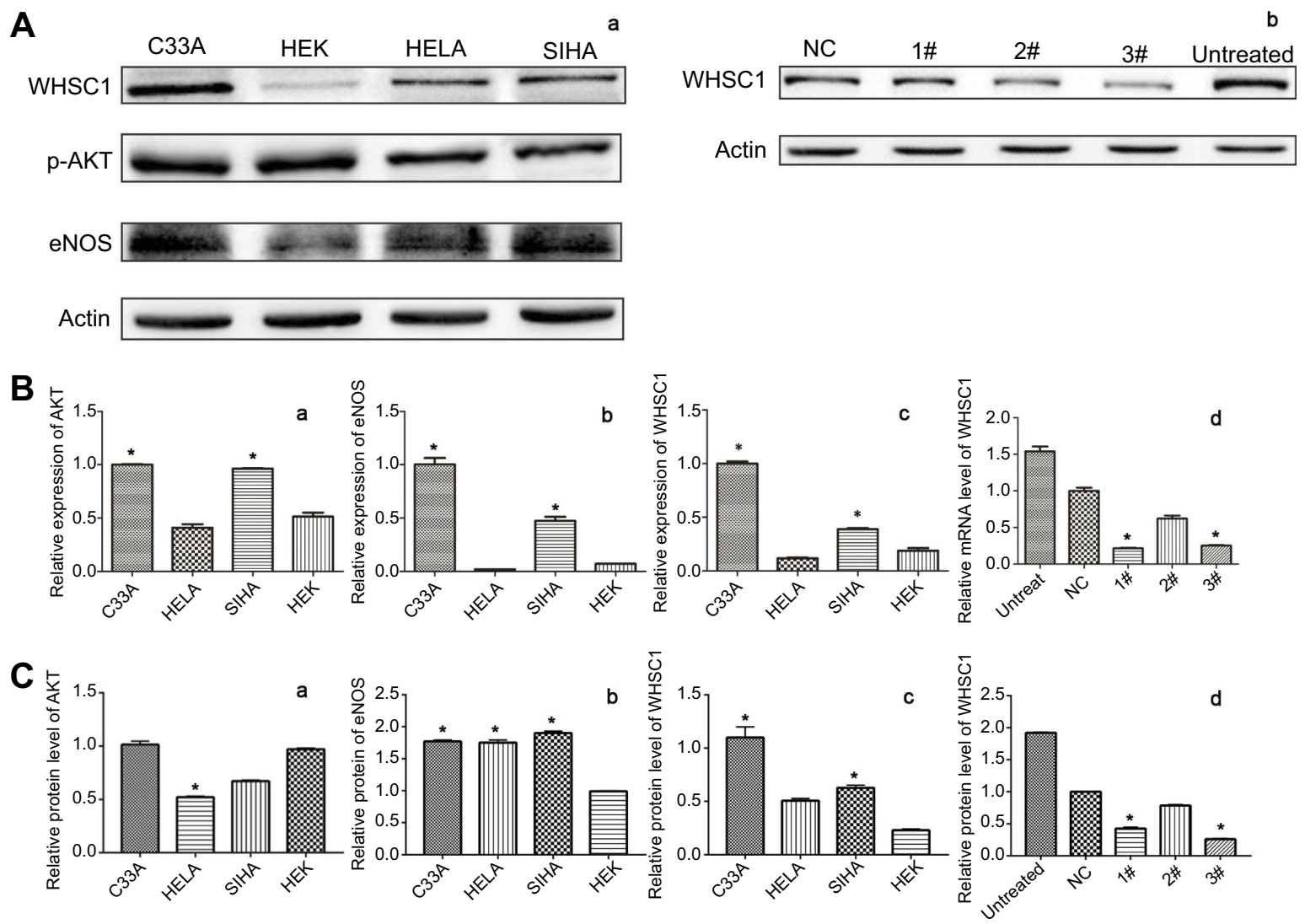

Figure 2 Expression levels of WHSCI in CC cells and its inhibition by siWHSCI in C33A cells. (A) WHSCI protein levels in cells were detected by Western blot: (a) WHSCI, AKT, and eNOS protein levels in C33A, HELA, SIHA, and HEK cells were detected by Western blot; (b) WHSCI protein levels were inhibited by siWHSCI in C33A cells. (B) The mRNA levels in cervical cells were detected by reverse transcription PCR and relative quantification analyzed by normalizing to actin mRNA: (a) AKT mRNA levels in C33A and SIHA were higher than those in HEK cells $(* P<0.05)$; (b) mRNA levels of eNOS in C33A and SIHA cells were higher than those in HEK cells $(* P<0.05)$; (c) WHSCI mRNA levels in C33A and SIHA were higher than those in HEK cells $(* P<0.05)$; (d) WHSCI mRNA levels were inhibited by siWHSCI-I\# and siWHSCI-3\# in C33A cells ( $* P<0.05)$, compared to those in untreated cells. (C) The protein levels in cells were detected by Western blot: (a) AKT protein levels in HELA cells were lower than those in HEK cells ( $\left.{ }^{*} P<0.05\right)$; (b) protein levels of eNOS in C33A, HELA, and SIHA cells were higher than those in HEK cells $\left({ }^{*} P<0.05\right)$; (c) WHSCI protein levels in C33A cells were higher than those in HEK cells $(* P<0.05)$; (d) WHSCI protein levels were significantly inhibited by siWHSCI-I\# and siWHSCI-3\# in C33A cells $(* P<0.05)$, compared to those in untreated cells.

Abbreviations: CC, cervical cancer; eNOS, endothelial nitric oxide synthase; HEK, human embryonic kidney; NC, normal control; si, small interfering; WHSCI, WolfHirschhorn syndrome candidate I.

WHSC1 expression, and that of AKT was slightly decreased (Figure 4B).

\section{Discussion}

Aberrant epigenetic changes are considered a hallmark of cancer. ${ }^{12}$ As a histone lysine methyltransferase enzyme involved in the regulation of epigenetic changes, WHSC1 is upregulated and acts as a tumor promotor in various human cancers. ${ }^{11,13-15}$ In the current study, we investigated the role of WHSC1 in CC and explored the underlying mechanism. We found that WHSC1 expression was remarkably increased in $\mathrm{CC}$ tissues and cell lines. Furthermore, we verified that WHSC1 promoted cell proliferation, migration, and invasion in CC. Therefore, our data suggest WHSC1 has a crucial role in the development of CC.
We report that WHSC1 expression was significantly increased in $\mathrm{CC}$ tissues; the strong positive staining in the tissue samples of $\mathrm{CC}$ further supported the results. Additionally, WHSC1 upregulation was positively correlated with the FIGO stage and differentiation, suggesting that WHSC1 might be a possible novel biomarker for poor prognosis of CC. Our results were consistent with those of other studies. ${ }^{16}$

NSD2 was initially described as a gene rearranged and linked to regulatory sequences of the immunoglobulin heavy chain gene in $\mathrm{t}(4 ; 14)$ multiple myeloma ${ }^{8,17}$ In a recent study, researchers reported that WHSC1 represents an "epigenetic hit," collaborating with PTEN loss to promote metastatic transformation. ${ }^{18}$ Nevertheless, only a few studies have explored the function of WHSC1 in the onset and development of CC. Toward that goal, we investigated the effect of 

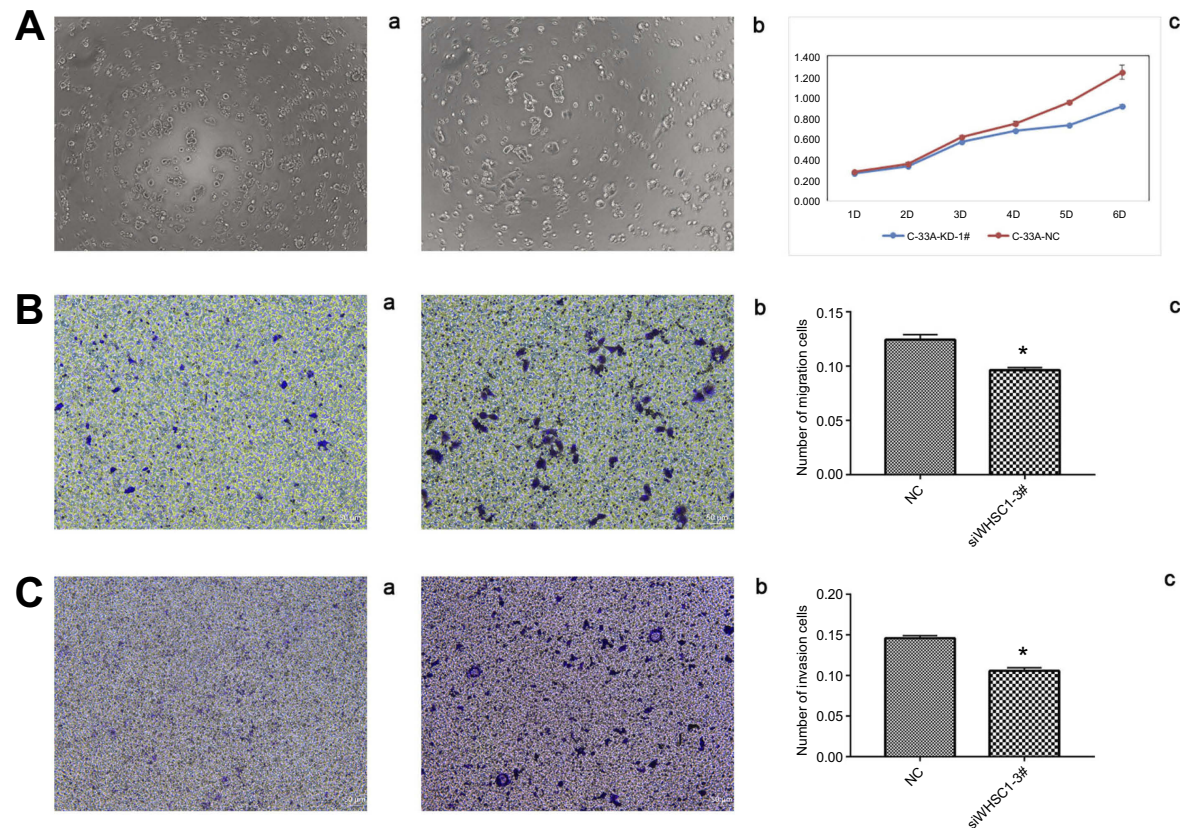

Figure 3 Effects of siWHSCI on cell growth, migration, and invasion of C33A cells. (A) Effects of WHSCl inhibition on C33A cell growth were detected by Cell Counting Kit-8 assay: (a) C33A-siWHSCI in 6 days $(\times 10)$; (b) C33A-NC in 6 days $(\times 10)$; (c) growth of C33A cells was inhibited by siWHSCI; $* P<0.05$, compared to that of siNCtreated cells. (B) Effects of WHSCI inhibition on the migration of C33A cells were detected by Transwell assay: (a) migration of C33A-siWHSCI cells ( $\times 10)$; (b) migration of C33A-NC cells $(\times 10)$; (c) inhibition of migration of C33A cells by siWHSCI; $* P<0.05$, compared to that of siNC-treated cells. (C) Effects of WHSCI inhibition on the invasion of C33A cells were detected by Transwell assay: (a) invasion of C33A-siWHSCI cells ( $\times 5)$; (b) invasion of C33A-NC cells $(\times 5)$; (c) inhibition of $\mathrm{C} 33 \mathrm{~A}$ cell invasion by siWHSCI; $* P<0.05$, compared to that of siNC-treated cells.

Abbreviations: NC, normal control; si, small interfering; WHSCI, Wolf-Hirschhorn syndrome candidate I.

A
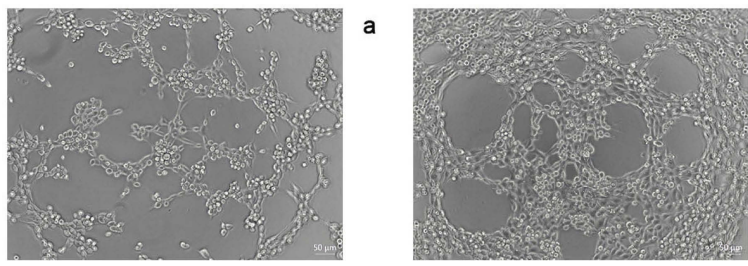

B

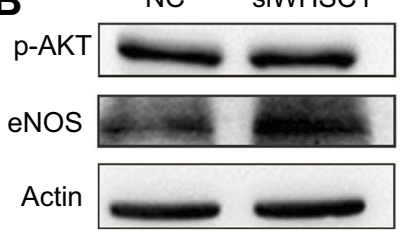

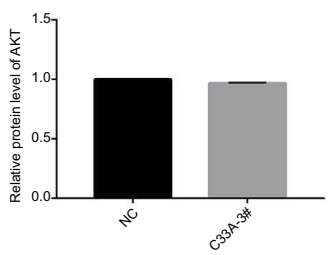

b
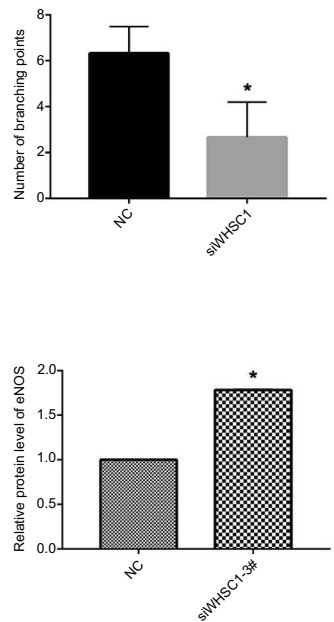

C

Figure 4 Effects of WHSCI inhibition on angiogenesis were detected by in-vitro tube formation assay and eNOS signaling pathway. (A) Effects of WHSCI inhibition on angiogenesis were detected by in-vitro tube formation assay: (a) angiogenesis of HUVECs was stimulated by the conditioned culture medium of C33A-siWHSCI ( $\times 10$ ); (b) angiogenesis of HUVECs was stimulated by the conditioned cultures media of C33A-siNC $(\times 10)$; (c) angiogenesis formation of HUVECs was inhibited by the culture medium of $\mathrm{C} 33 \mathrm{~A}$-siWHSCl cells; $* P<0.05$, compared to that in siNC cells. (B) Effect of siWHSCI on eNOS signaling pathway: (a) the effect of WHSCI knockdown on the expression of AKT and eNOS was assessed by Western blot in C33A cells; (b) AKT protein levels were not significantly inhibited by siWHSCI in C33A cells, compared to those in untreated cells; (c) the protein levels of eNOS were significantly inhibited by siWHSCI in C33A cells, ${ }^{* P<0.05}$ vs untreated cells.

Abbreviations: eNOS, endothelial nitric oxide synthase; NC, normal control; si, small interfering; WHSCI, Wolf-Hirschhorn syndrome candidate I.

WHSC1 knockdown on proliferation, invasion, and migration of C33A cells. Results showed that C33A cell proliferation, migration, and invasion were significantly decreased when WHSC1 was silenced by siRNA. In addition, tube formation of HUVECs was repressed after knockdown of
WHSC1 in C33A cells. These results suggest that siWHSC1 may suppress tumor metastasis by inhibiting tumor cell proliferation, invasion, migration, and tumor angiogenesis. The results suggest that WHSC1 may become a new therapeutic target for CC. 
The family of nitric oxide synthases (NOS) comprises inducible NOS, eNOS, and neuronal NOS. Within mammalian cells, a family of NOS enzymes has been shown to be able to generate nitric oxide (NO). The free radical gas NO acts as a novel transcellular messenger molecule in many key physiological and pathological processes. ${ }^{19}$ High levels of NOS expression (eg, generated by activated macrophages) seem to be cytostatic or cytotoxic for tumor cells, whereas low-level activity may have the opposite effect and promote tumor growth. ${ }^{20}$ In our study, we found that expression of eNOS in C33A cells was further elevated by WHSC1 knockdown. We speculated that WHSC 1 may affect the expression of $\mathrm{NO}$ in the microenvironment of CC by regulating the NOS signaling pathway, thereby affecting the proliferation, migration, and invasion of cancer cells and angiogenesis of HUVECs.

\section{Conclusion}

Through these results, we speculate that WHSC1 might play an oncogenic role in $\mathrm{CC}$ and may be a potential therapeutic target for the same, and WHSC1 may achieve its role in promoting tumor progression through regulation of the eNOS signaling pathway. However, our results are limited to the level of in vitro experiments, and we will further verify them in animal experiments in the next study.

\section{Acknowledgments}

The authors are very grateful to Professor Lixue Chen of the Clinical Experimental Center of the First Affiliated Hospital of Chongqing Medical University for his careful guidance during the study.

\section{Disclosure}

The authors report no conflicts of interest in this work.

\section{References}

1. Torre LA, Bray F, Siegel RL, et al. Global cancer statistics 2012. CA Cancer J Clin. 2015;65(2):87-108. doi:10.3322/caac.21294

2. Denny L, de Sanjose S, Mutebi M, et al. Interventions to close the divide for women with breast and cervical cancer between low-income and middle-income countries and high-income countries. Lancet. 2017;389 (10071):861-870. doi:10.1016/S0140-6736(16)31795-0

3. Maleszewska M, Wojtas B, Kamińska B. Deregulation of epigenetic mechanisms in cancer. Postepy Biochem. 2018;64(2):148-156. doi:10.18388/pb.2018_125

4. Morishita M, Di Luccio E. Cancers and the NSD family of histone lysine methyltransferases. Biochim Biophys Acta. 2011;1816 (2):158-163. doi:10.1016/j.bbcan.2011.05.004
5. Bennett RL, Swaroop A, Troche C, Licht JD. The role of nuclear receptor-binding SET domain family histone lysine methyltransferases in cancer. Cold Spring Harb Perspect Med. 2017;7(6): a026708. doi:10.1101/cshperspect.a026708

6. Keats JJ, Maxwell CA, Taylor BJ, et al. Overexpression of transcripts originating from the MMSET locus characterizes all $\mathrm{t}(4 ; 14)(\mathrm{p} 16$; q32)-positive multiple myeloma patients. Blood. 2005;105 (10):4060-4069. doi:10.1182/blood-2004-09-3704

7. Santra M, Zhan F, Tian E, et al. A subset of multiple myeloma harboring the $\mathrm{t}(4 ; 14)(\mathrm{p} 16 ; \mathrm{q} 32)$ translocation lacks FGFR3 expression but maintains anIGH/MMSET fusion transcript. Blood. 2003;101(6):2374-2376. doi:10.1182/blood-2002-09-2801

8. Chesi M, Nardini E, Lim RS, et al. The t $(4 ; 14)$ translocation in myeloma dysregulates both FGFR3and a novel gene, MMSET, resulting in IgH/MMSET hybrid transcripts. Blood. 1998;92 (9):3025-3034.

9. Hudlebusch HR, Santoni-Rugiu E, Simon R, et al. The histone methyltransferase and putative oncoprotein MMSET is overexpressed in a large variety of human tumors. Clin Cancer Res. 2011;17 (9):2919-2933. doi:10.1158/1078-0432.CCR-10-1302

10. Ezponda T, Popovic R, Shah MY, et al. The histone methyltransferase MMSET/WHSC1 activates TWIST1 to promote an epithelial-mesenchymal transition and invasive properties of prostate cancer. Oncogene. 2013;32(23):2882-2890. doi:10.1038/ onc. 2012.297

11. Saloura V, Cho HS, Kiyotani K, et al. WHSC1 promotes oncogenesis through regulation of NIMA-related kinase-7 in squamous cell carcinoma of the head and neck. Mol Cancer Res. 2015;13(2):293-304. doi:10.1158/1541-7786.MCR-14-0292-T

12. Flavahan WA, Gaskell E, Bernstein BE. Epigenetic plasticity and the hallmarks of cancer. Science. 2017;357:6348. doi:10.1126/science. aal2380

13. Suhaimi SS, Ab Mutalib NS, Khor SS, et al. Targeted next-generation sequencing identifies actionable targets in estrogen receptor positive and estrogen receptor negative endometriod endometrial cancer. Front Pharmacol. 2018;9:750. doi:10.3389/ fphar.2018.00750

14. Dong P, Xiong Y, Yue J, Hanley SJB, Watari H. miR-34a, miR-424 and miR-513 inhibit MMSET expression to repress endometrial cancer cell invasion and sphere formation. Oncotarget. 2018;9 (33):23253-23263. doi:10.18632/oncotarget.25298

15. Hudlebusch HR, Skotte J, Santoni-Rugiu E, et al. MMSET is highly expressed and associated with aggressiveness in neuroblastoma. Cancer Res. 2011;71(12):4226-4235. doi:10.1158/0008-5472.CAN10-3810

16. Yin Z, Sun Y, Ge S, Sun J. Epigenetic activation of WHSC1 functions as an oncogene and is associated with poor prognosis in cervical cancer. Oncol Rep. 2017;37(4):2286-2294. doi:10.3892/ or. 2017.5463

17. Stec I, Wright TJ, van Ommen GJ, et al. WHSC1, a $90 \mathrm{~kb}$ SET domain-containing gene, expressed in early development and homologous to a drosophila dysmorphy gene maps in the Wolf-Hirschhorn syndrome critical region and is fused to $\operatorname{IgH}$ in $\mathrm{t}(4 ; 14)$ multiple myeloma. Hum Mol Genet. 1998;7(7):1071-1082.

18. Li N, Xue W, Yuan $\mathrm{H}$, et al. AKT-mediated stabilization of histone methyltransferase WHSC1 promotes prostate cancer metastasis. J Clin Invest. 2017;127(4):1284-1302. doi:10.1172/ JCI91144

19. Moncada S, Palmer RM, Higgs EA. Nitric oxide: physiology, pathophysiology, and pharmacology. Pharmacol Rev. 1991;43:109-142.

20. Xu W, Liu LZ, Loizidou M, Ahmed M, Charles IG. The role of nitric oxide in cancer. Cell Res. 2002;12(5-6):311-320. doi:10.1038/sj. cr.7290133 


\section{Supplementary materials}

Table SI The sequences of siRNA oligos against WHSCI

\begin{tabular}{|l|l|}
\hline WHSCI siRNA & Sequence \\
\hline WHSCI-siRNA-I-F & 5'-TGCTGTGCTGAGGAACACAGAACACTGTTTTGGCCACTGACTGACAGTGTTCTGTTCCTCAGCA-3' \\
WHSCI-siRNA-I-R & 5'- CCTGTGCTGAGGAACAGAACACTGTCAGTCAGTGGCCAAAACAGTGTTCTGTGTTCCTCAGCAC -3' \\
WHSCI-siRNA-2-F & 5'- TGCTGATGCAAGGCCACCAAGGGTAAGTTTTGGCCACTGACTGACTTACCCTTTGGCCTTGCAT -3' \\
WHSCI-siRNA-2-R & 5'-CCTGATGCAAGGCCAAAGGGTAAGTCAGTCAGTGGCCAAAACTTACCCTTGGTGGCCTTGCATC-3' \\
WHSCI-siRNA-3-F & 5'- TGCTGTTTCGTTGCTGCCTGGCTCTTGTTTTGGCCACTGACTGACAAGAGCCACAGCAACGAAA -3' \\
WHSCI-siRNA-3-R & 5'- CCTGTTTCGTTGCTGTGGCTCTTGTCAGTCAGTGGCCAAAACAAGAGCCAGGCAGCAACGAAAC -3' \\
\hline
\end{tabular}

Abbreviations: siRNA, small interfering RNA; WHSCI, Wolf-Hirschhorn syndrome candidate I.

Table S2 The sequences of primers used

\begin{tabular}{|l|l|l|}
\hline Primer & Sequence & Size (bp) \\
\hline WHSCI-F & ATTTAGCATCAAGCAGAGTCCC & 291 \\
WHSCI-R & CGCAGTTTGGCATCGTGT & \\
AKT-F & CGAGGTGCTGGAGGACAAT & 285 \\
AKT-R & CACACGATACCGGCAAAGAA & \\
ENOS-F & GCCGAGGAGACTTCCGAA & 270 \\
ENOS-R & CCAGGGCTGCAAACCACT & \\
\hline
\end{tabular}

Abbreviations: ENOS, endothelial nitric oxide synthase; WHSCI, Wolf-Hirschhorn syndrome candidate I.

\section{Publish your work in this journal}

OncoTargets and Therapy is an international, peer-reviewed, open access journal focusing on the pathological basis of all cancers, potential targets for therapy and treatment protocols employed to improve the management of cancer patients. The journal also focuses on the impact of management programs and new therapeutic agents and protocols on patient perspectives such as quality of life, adherence and satisfaction. The manuscript management system is completely online and includes a very quick and fair peer-review system, which is all easy to use. Visit http://www.dovepress.com/ testimonials.php to read real quotes from published authors. 